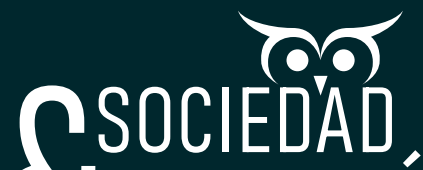 ¿ECONOMÍA
}

N

$\mathbf{N}^{\circ} 44$

Sep - dic 2021

\section{Competencia monopolística y externalidades en el criptomercado de drogas} Monopolistic Competition and Externalities in The Drug Crypto Market

\section{Leonardo Raffo'}

Universidad del Valle, Cali, Colombia

@ leonardo.raffo@correounivalle.edu.co

(1D) https://orcid.org/0000-0001-6087-535X

\section{Diego Gómez-Calderón ${ }^{2}$}

Universidad Nacional Abierta y a Distancia, Cali, Colombia

심iegoj.gomez@unad.edu.co

(D) https://orcid.org/0000-0002-2619-5673

Carolina Ramírez-Andrade ${ }^{3}$

Consultora independiente, Cali, Colombia

(2) caroramirezandrade@gmail.com

(D) https://orcid.org/0000-0002-8693-4984

Recibido: 19-01-2021

Aceptado: 01-07-2021

Publicado: 15-09-2021

1 Magíster en Economía Aplicada.

2 Magíster en Economía Aplicada.

3 Magíster en Economía Aplicada. 


\title{
Resumen
}

El propósito de la investigación es explicar el funcionamiento y dinámica del criptomercado de drogas. Con ese propósito se desarrolló un modelo de competencia monopolística con externalidades en red basado en los trabajos Belleflamme y Peitz (2010) y Dixit y Stiglitz (1977). La principal predicción del modelo es que las políticas de interdicción en estos mercados tienden a ser contrarrestadas por la entrada de nuevos vendedores, y la resultante apertura de nuevas páginas web en la red oscura. Otro hallazgo es que, debido a la presencia de efectos de externalidad en red en el consumo de drogas online, el endurecimiento de las políticas de interdicción produce un efecto de retroalimentación que lleva a que a un mayor número efectivo de consumidores decida adquirir los dispositivos para acceder a las plataformas de drogas en la web oscura y, así, compre drogas ilegales.

Palabras clave: criptomercados; drogas ilícitas; economía del crimen; competencia monopolística; externalidades en red.

Clasificación JEL: K42, L81, L86, D43, C72, D85.

\begin{abstract}
The research purpose is to explain the functioning and dynamics of the crypto drug market. To that end, a model of monopolistic competition with network externalities was developed based on the works Belleflamme \& Peitz (2010) and Dixit \& Stiglitz (1977). The model's main prediction is that interdiction policies in these markets tend to be counteracted by the entry of new sellers and the resulting opening of new websites in the dark web. A further finding is that, due to the presence of network externality effects in online drug use, the tightening of interdiction policies produces a feedback effect that leads to a greater number of effective consumers deciding to acquire the devices to access drug platforms on the dark web and, thus, to purchase illegal drugs.

Keywords: crypto markets; illicit drugs; economics of crime; monopolistic competition; network externalities. JEL Classification: K42, L81, L86, D43, C72, D85.
\end{abstract}

\section{Financiación}

Este artículo hace parte de una investigación sobre el narcotráfico y los mercados ilegales desarrollada por los autores. Se basa en parte en la tesis de maestría de Carolina Ramírez, la cual fue distinguida como Tesis Meritoria. También se apoya en la investigación doctoral de Leonardo Raffo.

\section{Agradecimientos}

Agradecemos los comentarios de los profesores Boris Salazar y Luis Eduardo Sandoval, así como los de dos evaluadores anónimos. Asimismo, agradecemos a los comentarios planteados por los asistentes del $\mathrm{V} \mathrm{Ne}$ twork de Microeconomía realizado el día 6 de mayo de 2021 en la Universidad Militar de la Nueva Granada.

\section{Conflicto de interés}

Los autores declaran no tener ningún conflicto de interés en la publicación de este artículo.

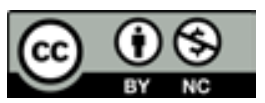

Este trabajo está bajo la licencía Atribución-No-Comercial 4.0 Internacional

¿Cómo citar este artículo?

Raffo, L., Gómez-Calderón, D. y Ramírez-Andrade, C. (2021). Competencia monopolística y externalidades en el criptomercado de drogas. Sociedad y economía, (44), e1 061 0962. https:// doi.org/10.25100/sye.v0i44.10962 


\section{Introducción}

Los criptomercados constituyen espacios de intercambio virtuales en donde bienes y servicios lícitos e ilícitos pueden ser transados con una mayor capacidad de anonimato que en los espacios transaccionales tradicionales (Décary-Hétu et al., 2016). Las transacciones ilegales que operan en los criptomercados, objeto del presente documento ${ }^{4}$, se realizan en la denominada Internet Oscura o Dark Web ${ }^{5}$, cuya característica fundamental es el anonimato de los agentes que transan en ella, el cual se logra gracias a los procesos de encriptación digital (Martin, 2014). Otra característica esencial de estos mercados es la posibilidad de transar bienes y servicios provenientes de países diversos, con destinos igualmente diversos y en muchos casos distantes. Se trata de mercados que han tendido a crecer y desarrollarse progresivamente durante las últimas décadas. Por ejemplo, para el periodo 2013-2015, estos facturaron tan solo en ventas de drogas ilícitas por lo menos una suma de 27 millones de dólares (The Economist, 2016).

Los vendedores de drogas ilícitas a través de los criptomercados tienden a enfrentar riesgos y retos muy diferentes a los de los mercados de drogas tradicionales en espacios físicos, debido a que el anonimato logrado por la encriptación digital permite reducir el número de intermediarios y contactos cara a cara (Décary-Hétu et al., 2016), alcanzando mayores estándares de seguridad y minimizando las dinámicas de violencia que se tienden a producir en los mercados tradicionales. El resultado de esta estructura tecnológica es la imposibilidad

4 En este trabajo se obvia el análisis de los criptomercados legales y los blockchain que los soportan, ya que se enfoca en el estudio del criptomercado específico de drogas ilegales. Por esta misma razón, tampoco se aborda el tema más general de las criptomonedas. Este es un tema muy interesante para ser desarrollado en próximos trabajos

5 La web oscura (también denominada como Dark Net) constituye una parte de la Deep Web o red profunda a la que únicamente se puede acceder mediante el uso de redes de servidores con varios niveles de encriptamiento. por parte de las autoridades de control de implementar las políticas de represión en la forma convencional. Como advierten DécaryHétu et al. (2016), los criptomercados alteran el riesgo de detección y arresto al proteger la identidad de los vendedores y, además, en ellos tienden a utilizarse criptomonedas como el Bitcoin, entre otras, las cuales potencian el anonimato aun en el pago del proceso transaccional (Martin, 2014).

En los criptomercados, los vendedores enfrentan dos tipos de riesgos distintos en diferentes etapas del proceso de comercialización de las sustancias ilícitas: por una parte, el riesgo constante de que sus páginas sean cerradas -como fue el caso de Silk Road en octubre de 2013-, al ser identificadas por las autoridades de control. En segundo lugar, el riesgo de que las drogas sean interceptadas antes de llegar a su destino. En consecuencia, en esta clase de mercados las políticas de interdicción se pueden desarrollar en cualquiera de estas dos etapas de comercialización de las drogas ilícitas.

El primer tipo de riesgo implica que un reto constante para los vendedores online de esta clase drogas sea contar con la habilidad y medios informáticos suficientes para abrir en poco tiempo nuevas páginas web. El segundo tipo de riesgo lleva a que un reto de los vendedores ilegales en criptomercados sea buscar que el envío de la mercancía sea lo más discreto y camuflado posible, borrando la huella del lugar de origen y de los remitentes. El resultado de esta forma de funcionamiento es que la probabilidad de incautación de las drogas tiende a ser menor que en los mercados tradicionales.

A pesar de la importancia creciente de los criptomercados, principalmente de las operaciones ilegales en dicho espacio virtual transaccional, hasta la fecha son muy escasos los trabajos teóricos que se han hecho para entender su funcionamiento -ver más adelante en la segunda sección-, por lo que es imprescindible preguntarse: ¿cuáles son los agentes que intervienen en este tipo de transacciones?, ¿qué interacciones se 
desenvuelven entre ellos?, ¿cómo influye la estructura tecnológica de la red oscura en las dinámicas en este mercado? Estas son preguntas que se intenta responder en el presente artículo, cuyo objetivo principal es entender cómo funcionan las operaciones de drogas ilegales en los criptomercados e identificar las principales dinámicas que determinan sus transacciones, tanto en el corto como en el largo plazo.

El presente artículo es la continuación de un trabajo previo de naturaleza descriptiva y conceptual sobre los criptomercados de drogas ilegales. Se desarrolla un modelo analítico que explica la dinámica de interacción entre traficantes y consumidores de drogas ilegales, a través de este nuevo espacio web transaccional. El marco teórico se centra en la economía del crimen y, en particular, en el estudio de los mercados ilegales. Dentro de este último conjunto de trabajos, se exploran algunos recientes que han abordado el tema de los criptomercados, una temática con escasa literatura hasta la fecha, pero con gran importancia ahora y en el futuro, debido al crecimiento vertiginoso del comercio online y, en particular, de los mercados que día tras día crecen y mutan en la Internet oscura.

La hipótesis de partida es que la estructura tecnológica y la reputación son determinantes de las decisiones de compra de drogas ilegales en los criptomercados. La metodología se centra en la construcción de un modelo analítico basado en el trabajo de Belleflamme y Peitz (2010), el cual a su vez se basa en el modelo canónico de Dixit y Stiglitz (1977). El modelo analiza las interacciones estratégicas entre los vendedores de dogas y los consumidores en la web oscura, en el contexto de una estructura de mercado con competencia monopolística. Investigaciones empíricas recientes han dado indicios de que los criptomercados constituyen espacios virtuales en donde confluye un número grande $-y$ potencialmente aún más grande- de vendedores (Dolliver, 2015; Décary-Hétu et al., 2016) más o menos "aislados entre sí", aunque "protegidos por una red virtual aparentemente anónima" (Dolliver, 2015 , p. 1122); y en donde, además, tiende a existir libre entrada y salida de vendedores, así como diferenciación horizontal y vertical de los productos ofrecidos online. No obstante, este trabajo se limita a examinar las consecuencias de la diferenciación horizontal de las drogas ofrecidas por página web. Estas son las características que hacen pensar que una aproximación analítica fecunda para entender el funcionamiento de este criptomercado es suponer que exhibe competencia monopolística, ya que es bien sabido que este tipo de estructuras de mercado se caracterizan por la presencia de un número grande de firmas -y un número potencialmente aún más alto de estas-, al igual que por la existencia de libre entrada y salida de las mismas ${ }^{6}$.

En la medida en que los modelos económicos logren acercarse a la realidad del funcionamiento de los criptomercados, especialmente a las transacciones de drogas ilícitas, será posible conocer el volumen de mercancías y el flujo de dinero que circulan en los mismos. De esta manera, las autoridades de control podrán tomar decisiones de política criminal efectivas para reducir el tráfico de bienes ilegales en este tipo de plataformas. En este sentido, este trabajo de investigación contribuye al análisis de la dinámica de los criptomercados, al desarrollar un modelo económico que revela las implicaciones de su estructura tecnológica y la reputación de los vendedores en las decisiones de compra en este tipo de mercados de drogas ilícitas.

Este documento se organiza de la siguiente manera: en la primera parte, luego de la presente

6 Desde luego, esto no implica que la única alternativa teórica para entender los criptomercados sea suponer competencia monopolística con diferenciación horizontal. En efecto, existen otras alternativas teóricas -como se menciona en la próxima sección-, no obstante, por parsimonia y evidencia empírica de carácter exploratoria -según los trabajos mencionadosesta elección teórica es una alternativa fecunda para entender de forma relativamente sencilla el funcionamiento de estos mercados, y constituye un buen punto de partida como modelo de línea de base para el desarrollo de futuras investigaciones en la materia. Pero aún queda mucho por pensar sobre los criptomercados. 
introducción, se exponen algunos conceptos fundamentales y la revisión de la literatura en la materia. En la segunda parte, se presentan los supuestos del modelo. En la tercera parte, se expone la solución del modelo. En la cuarta, se muestran los principales hallazgos encontrados. En la quinta y sexta parte, se discuten algunas implicaciones para la política antidrogas en el contexto de los criptomercados. Por último, se presentan algunas conclusiones y consideraciones.

\section{Hacia un modelo analítico del criptomercado de drogas ilícitas}

Actualmente existe una amplia literatura sobre la economía del crimen. Tanto los enfoques teóricos como los empíricos se han desarrollado a lo largo de los años para comprender mejor los costos y beneficios de la delincuencia (Garoupa, 1997; Polinsky y Shavell, 2000). En particular, la interacción entre el mercado de la delincuencia y los otros efectos de equilibrio general son cruciales para generar políticas más efectivas (Ballester et al., 2006; 2010).

En el caso de las operaciones en el criptomercado de drogas ilícitas, la oferta está constituida por los administradores, moderadores y vendedores. Sin embargo, este análisis considera la fusión del rol de administrador y vendedor. Adicionalmente, el rol del moderador se excluye del análisis, dado que este se limita al mantenimiento de la página web y no incide en las decisiones de estructura tecnológica, el establecimiento de las reglas del juego entre usuarios ni en las transacciones. Por su parte, la demanda está constituida por los consumidores de drogas ilícitas. Los traficantes, entendidos como administradores/vendedores, manejan las relaciones y deciden de manera no cooperativa cuánto esfuerzo de delincuencia ejercerán en el espacio de mercado.

La estructura de las redes criminales que soportan esta clase de mercados es distinta a la de los mercados de drogas tradicionales que funcionan como redes jerárquicas o de jerarquía agrupada (Raffo y Segura, 2015; Raffo y
Gómez, 2017; Raffo y Segura 2018). Estas, en cambio, se han adaptado a las condiciones del entorno, tanto por los consumidores como por los agentes represores (Heber, 2009; Malm y Bichler, 2011). En concreto, se trata de redes criminales que han evolucionado incorporando una serie de avances relacionados con las tecnologías de información y las redes de comunicación en línea.

El criptomercado de drogas ilícitas puede abordarse desde el enfoque de competencia monopolística. Lo anterior se sustenta, aparte de lo planteado atrás, al tener en cuenta que dicho mercado es controlado por los administradores, a través del cual interactúan múltiples vendedores -con una 'vida útil' promedio de un año, según Janetos y Tilly (2017)-, que realizan operaciones de drogas ilegales de forma anónima, sin que exista conocimiento de un control dominante de alguno de ellos, generando particularidades en las condiciones de navegabilidad de las páginas.

Bajo este criterio funcional del mercado, se debería construir un equilibro a dos lados (Two Sided Markets) o realizar un análisis de mercados bilaterales (Tirole y Rochet, 2003), es decir, el administrador define una estructura de precios y cantidades óptimas para los vendedores, y otras condiciones de mercado para los compradores, generando su ganancia del diferencial de precios. Dado que la investigación se centra en la estructura de equilibro del criptomercado de drogas ilegales, es válida la integración de los roles: administrador y vendedor. Esta distinción simplemente expresa una particularidad tecnológica de los mercados que utilizan plataformas web para su operación.

Estos vendedores transan productos que se agrupan en categorías como marihuana, cocaína, MDMA (droga sintética que produce efectos estimulantes y alucinógenos, más comúnmente conocida como "éxtasis"), LSD, heroína, entre otros. Aunque los diferentes menús de drogas ofrecidos por cada vendedor pueden ser similares respecto a los tipos genéricos de sustancias ofrecidas, estos se preocupan por dar a conocer aspectos diferenciadores de sus 
productos, como información de la composición del producto, porcentaje de pureza de la droga, tiempo de entrega y forma de pago, entre otros. Esta diferenciación en el producto y en el servicio se ve reflejada en la reputación que los compradores dan a los vendedores al final de la transacción y, a su vez, les permite a los vendedores atraer nuevos clientes y fidelizar a los que ya tienen (Hardy y Norgaard, 2015).

En el presente análisis, el traficante decide la estructura tecnológica que le permitirá generar los diferenciadores de navegabilidad de las páginas. Esta estructura permitirá brindar una mejor experiencia en la navegación de los usuarios, facilitando que los compradores puedan acceder a búsquedas sencillas de sus productos y que la plataforma arroje listados de productos con la mayor información disponible, ligados a la reputación de cada página.

La estructura tecnológica con la que un traficante (administrador/vendedor) construye o modifica su criptomercado determina, entre otras cosas, el nivel de encriptamiento de las transacciones, el tipo de información que él podrá incluir en su perfil, el número y calidad de fotos de los productos, los foros de discusión disponibles y sus tiempos de respuesta, los mecanismos de pago disponibles, la forma de solución de disputas, etc.

La diferenciación vertical relacionada con la estructura tecnológica permite a los traficantes establecer el precio de sus productos (Hardy y Norgaard, 2015), sacando en el largo plazo a los vendedores con reputación baja (Janetos y Tilly, 2017). Por esta razón, los traficantes, desde su rol de administrador, deberán mejorar constantemente la estructura tecnológica de su página, lo cual permitirá mejorar las condiciones de navegabilidad, seguridad y diferenciales de servicio para las transacciones, potenciando su rentabilidad.

Si bien las operaciones de drogas ilícitas en el criptomercado implican condiciones tecnológicas como punto de partida para operar (requieren altos niveles de capital humano especializado, así como disponibilidad y dominio de determinados ambientes técnicos y tecnológicos), una vez el traficante cuente con los equipos técnicos y el conocimiento tecnológico de dicho mercado, existe libre entrada y salida de firmas ${ }^{7}$. En este sentido, el siguiente modelo de competencia monopolística, con externalidades en red, analiza la dinámica del criptomercado a partir de la interacción entre traficantes y compradores de drogas ilegales, a través de estructuras tecnológicas que les permiten transar estos bienes en la web oscura y, de paso, contribuyen a definir la reputación de las páginas web. El presente modelo se basa en el modelo de competencia monopolística con externalidades en red expuesto por Belleflamme y Peitz (2010), en el capítulo 20 de su texto, quienes a su vez se basan en el modelo canónico de Dixit y Stiglitz (1977). En Raffo (2016), se hace una exposición detallada del primer modelo.

\section{El modelo}

El modelo define una transacción de drogas ilícitas en el criptomercado como la combinación de uno de los diferentes tipos de droga disponibles en una página web específica; es decir, la variedad del producto es la relación dual droga-página web. La definición anterior permite fusionar el rol del administrador y del moderador en el rol del vendedor (traficante), para explicar la interacción con el consumidor, y las dinámicas de mercado subyacentes. Existen otras alternativas de modelación, una de ellas se centra en la interacción de los agentes de la cadena de abastecimiento. En esta alternativa, la diferenciación de los roles de administrador, moderador y vendedor es fundamental. Lo anterior evidencia las múltiples posibilidades de modelación que puede tener un criptomercado de drogas ilegales.

Cada página web es administrada por un único traficante, quien provee a través de ella di-

7 En la práctica, tener altos niveles de capital humano, así como disponibilidad y dominio de ciertos ambientes técnicos y tecnológicos, en realidad no constituye una barrera de entrada para entrar a los criptomercados, ya que el número de trabajadores activos con estas características y con acceso a las tecnologías requeridas es alto y creciente. 
versas variedades de drogas ilegales. Es decir, una variedad de marihuana (por ejemplo) puede venderse en varias páginas web, pero cada página web representará solo a un traficante (ver Figura 1). Es importante aclarar que este tipo de modelación se centra en la diferenciación que recrea el criterio reputacional de cada página web respecto a la droga, es decir, las páginas web pueden vender los mismos tipos de drogas a través de alternativas tecnológicas diferenciadoras. Otro tipo de modelación podría basarse en la diferenciación del producto, lo cual tiene que ver con particularidades físicas del bien.

Figura 1. Esquema de modelo para el criptomercado de drogas ilegales

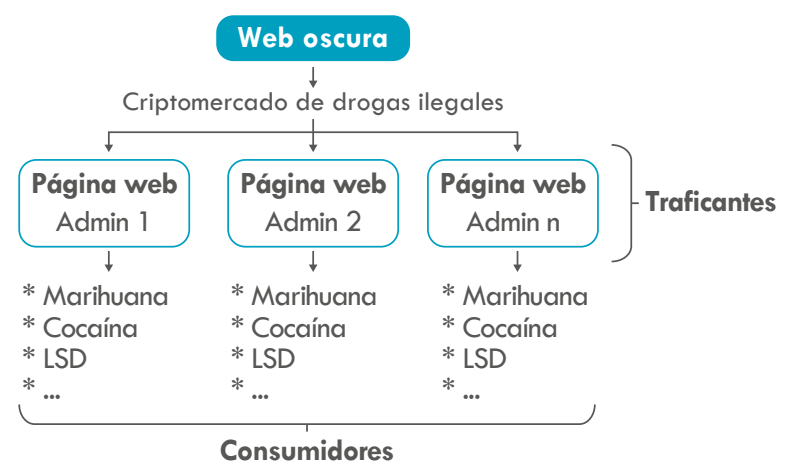

Fuente: elaboración propia a partir de la investigación realizada.

Según lo anterior, un bien $j$ debe entenderse como la combinación droga-página web, por ende, existen tantos bienes como cambios existan en la variedad de la droga, en la variedad de la página, o en ambos. En ese sentido, se supone que existe diferenciación horizontal en las ventas de drogas en la red oscura, siendo cada una de las variedades las distintas combinaciones droga-página web.

Adicionalmente, se tienen $n$ consumidores con preferencias definidas sobre los elementos técnicos para acceder a la web oscura -un encriptador de rutas (TOR-VPN) y un encriptador de mensajes (PGP)-; el criptomercado (páginas web donde venden drogas ilegales) y un bien numerario externo, ofrecido en un mercado competitivo. Debido a su estructu- ra secuencial en tres etapas distintas (conectadas entre sí), el modelo se puede concebir como un juego secuencial. El orden temporal del juego es:

\section{a. Primera etapa:}

Los consumidores eligen el nivel agregado de gasto en variedades de drogas-páginas web y deciden si adquieren los dispositivos técnicos básicos para acceder a la web oscura y al criptomercado de drogas ilegales -según el nivel de seguridad que requieran-, antes de conocer el número total de páginas web que puedan estar abiertas en el segundo periodo.

\section{b. Segunda etapa:}

Los traficantes (administrador/vendedor) observan la decisión de adopción de los consumidores y proporcionan $m$ variedades de páginas web con variables de diferenciación (tipo de producto, nivel de pureza, tiempo de entrega, servicio al cliente y calificación del servicio, entre otros) en el segundo periodo.

\section{c. Tercera etapa:}

Los consumidores eligen los niveles óptimos de las diferentes variedades de drogas ilegales droga-página web $\left(C_{j}\right)$.

La web oscura configura un espacio de mercado alternativo a las transacciones de drogas ilícitas, las cuales se desarrollan principalmente en un territorio físico. El carácter finito del territorio establece la imposición de barreras de entrada en los mercados convencionales de drogas ilícitas, configurando una estructura de mercados oligopólica. Dado el carácter siempre potencialmente creciente de la web oscura, por la posibilidad de entrada sin restricciones de nuevos vendedores potenciales al mercado, la imposición de barreras de entrada no es factible. Dicho esto, como ya se argumentó atrás, la estructura de mercados subyacente al criptomercado de drogas ilegales se asemeja a la competencia monopolística, ya que el espacio transaccional son sitios virtuales que definen una red en sí misma, y es a partir de la sofisticación en la disponibilidad de variables diferenciadoras en las páginas web que se logra establecer un nivel reputacional que dinamice las ventas de drogas ilegales. 
La reputación de una página web está dada por el nivel de satisfacción de los consumidores, que a su vez está relacionado con los aspectos tecnológicos (nivel de seguridad de la página, interacción con el comprador a través de secciones de comentarios y preguntas, disponibilidad de fotografías reales del producto, etc.) y con el modelo de negocio (tiempo de la transacción y entrega efectiva del bien ilegal, en las condiciones acordadas entre las partes). Dicha reputación define el efecto de la diferenciación del producto, respecto a las particularidades de la página, lo cual se retroalimenta por una estructura de externalidades en red de la demanda.

Aunque no existe una dinámica de interdependencia estratégica entre las firmas en los mer- cados de competencia monopolística, por su estructura secuencial, el modelo puede concebirse como un juego dinámico que se resuelve por inducción hacia atrás. Lo anterior permite analizar los tres espacios esenciales que configuran las transacciones en el criptomercado de drogas ilegales.

Existen $n$ consumidores potenciales que pueden convertirse en consumidores online cuando deciden adquirir los dispositivos técnicos necesarios para acceder a la Deep Web y a la Dark Web. La función de utilidad de los consumidores es del tipo cuasilineal, con un componente que entra linealmente en esta (el numerario) y un bien compuesto de tipo CES, que capta el consumo de las diferentes variedades de droga-página web:

$$
\mathcal{U}=C_{0}+\phi\left[\left(\int_{0}^{m} C_{j}^{\rho} d_{j}\right)^{1 / \rho}\right]^{\beta} \quad ; \quad 0<\rho<1 \wedge \beta<\rho
$$

En donde $C_{0}$ es la cantidad del bien numerario demandada por cada consumidor de drogas. El numerario representa un bien a la Hicks-Hansen que captura el resto de bienes que puede consumir cada uno de ellos. $\phi$ constituye un parámetro positivo que capta el tipo de consumidor al establecer un sesgo específico hacia el consumo de drogas ilegales. $C_{j}$ representa la cantidad de variedad de droga por página web consumida por cada agente; por ejemplo, cannabis orgánico en la página Smokeables. Siguiendo a Dixit y Stiglitz (1977), $Z \equiv\left(\int_{0}^{m} C_{j}^{\rho} d_{j}\right)^{1 / \rho}$ puede concebirse como un bien compuesto $Z$, el cual corresponde a un índice de bienes de consumo, en este caso de variedades de droga-página web, que se diferencian horizontalmente entre sí, en el contexto de una sola industria con competencia monopolística.

Con el tipo de preferencias expresadas en la función de utilidad, puede probarse que la elasticidad de sustitución en una transacción en una página web $j$ (combinación de droga-página web) y una $k$ es $\theta=\frac{1}{1-\rho}$, y como $0<\rho<1$, entonces $\theta>1$, lo cual garantiza que se trata de bienes buenos sustitutos entre sí. Adicional- mente, como $\beta<\rho$, la utilidad marginal de una variedad extra de páginas web es decreciente.

Los consumidores están dotados con un nivel de ingreso $\hat{l}$, el cual pueden gastar en tres tipos de bienes distintos: en primer lugar, en el bien externo (el numerario, $C_{0}$ ), cuyo precio es 1 . En segundo lugar, en todo el conjunto de elementos técnicos básicos para el acceso a la web oscuracriptomercado al precio $P_{B}$, el cual se trata como un único bien de carácter unitario ${ }^{8}$. $Y$, en tercer lugar, en el conjunto de combinaciones de drogas-página web disponibles en la web oscura, cada una al precio $P_{j}$. Sea $E=\int_{0}^{m} P_{j} C_{j} d_{j}$, el gasto total de cada consumidor en las diferentes combinaciones droga-página web disponibles en la web oscura. La restricción presupuestaria queda entonces como

$$
\hat{I}=C_{0}+P_{B}+E
$$

8 Solo puede adquirirse una unidad de este conjunto de elementos técnicos que se concibe como un bien tecnológico expresado en una combinación de hardware, software y acceso a Internet. 
Desde el lado de la oferta, los traficantes operan con rendimientos crecientes a escala. El costo marginal de producción es constante e igual a $b$. Adicionalmente, las firmas enfrentan un costo fijo $F$. Por lo tanto, las funciones de costos $\left(B_{j}\right)$ están dadas por:

$$
B_{j}\left(q_{j}\right)=b q_{j}+F
$$

\section{Solución del modelo}

Dado que el modelo se resuelve por inducción hacia atrás, su solución comienza por la tercera etapa del juego:

\section{a. Tercera etapa:}

El problema que resuelven los consumidores en la tercera etapa es:

$$
\begin{aligned}
& \operatorname{Max} \mathcal{U}=C_{0}+\phi\left[\left(\int_{0}^{m} C_{j}^{\rho} d_{j}\right)^{1 / \rho}\right]^{\beta} \\
& \forall \mathrm{j} \in[0, m] \quad \text { s.a. } \hat{\mathrm{I}}=C_{0}+P_{B}+E
\end{aligned}
$$

Según lo anterior, el problema se resuelve planteando el siguiente Lagrangiano:

$$
\begin{gathered}
\mathrm{L}_{\left\{C_{j}\right\}}=C_{0}+\phi\left[\left(\int_{0}^{m} C_{j}^{\rho} d_{j}\right)^{1 / \rho}\right]^{\beta}+\lambda[\hat{\mathrm{I}} \\
\left.-C_{0}-P_{B}-E\right] ; \text { en donde } E=\int_{0}^{m} P_{j} C_{j} d_{j}
\end{gathered}
$$

Las condiciones de primer orden (C.P.O.) son:

$$
\begin{gathered}
\frac{\partial L}{\partial C_{i}}=0 ;\left(\frac{\beta}{\rho}\right) \phi\left(\int_{0}^{m} C_{j}^{\rho} d_{j}\right)^{(\beta / \rho)-1} \rho C_{j}^{\rho-1} \\
-\lambda p_{j}=0 \forall j \epsilon[0, m] \\
\frac{\partial L}{\partial \lambda_{i}}=0 ; \quad \hat{\mathrm{I}}-C_{0} P_{B}+E=0
\end{gathered}
$$

Dado que $(0<\rho<1) \Lambda(\beta<\rho)$, la segunda derivada del Lagrangiano con respecto a $C_{i}^{*}$ es negativa $\forall$ $j \in[0, m] i$, por lo que $\forall j \in[0, m], C_{i}^{*}$ es un máximo.

De la ecuación [5] se infiere que la demanda relativa para dos variedades $j$ y $k$ corresponde $a$

$$
\frac{C_{j}}{C_{k}}=\left(\frac{P_{j}}{P_{k}}\right)^{\frac{1}{\rho-1}}
$$

De aquí puede probarse que la elasticidad de sustitución entre cualquier par de variedades-páginas web es $\theta=\frac{1}{(1-\rho)}>1$.

Tras un poco de álgebra puede probarse que la demanda Marshalliana individual de $j$ es

$$
C_{j}=Z\left(\frac{P_{d}}{P_{j}}\right)^{\frac{1}{1-\rho}} \text {; }
$$

En donde $P_{d}=\left[\int_{0}^{m} P_{k}^{-\frac{\rho}{1-\rho}} d_{k}\right]^{-\frac{(1-\rho)}{\rho}}$ corresponde al índice de precios dual de $Z$.

Este resultado se puede formalizar a través de la siguiente proposición:

Proposición 1: La demanda marshalliana individual de una variedad de droga en una página web viene dada por $C_{j}=Z\left(\frac{P_{d}}{P_{j}}\right)^{\frac{1}{1-\rho}}$.

Prueba: De [7] $P_{k}\left(\frac{C k}{C j}\right)=\frac{P_{k} P_{k}^{-\frac{1}{1-\rho}}}{P_{j}^{-\frac{1}{1-\rho}}}$; integrando a ambos lados entre 0 y $m$ se obtiene $\int_{0}^{m} P_{k} \frac{C k}{c_{j}} \quad d_{k}=\int_{0}^{m} \frac{P_{k}{ }^{-\frac{\rho}{1-\rho}}}{P_{j}-\frac{1}{1-\rho}} d_{k} ;$

resolviendo para $C_{j}$, se llega a $C_{j}=$ $\frac{\int_{0}^{m} P_{k} c_{k} d_{k} P_{j}^{-\frac{1}{1-\rho}}}{\int_{0}^{m} P_{k}{ }^{-\frac{\rho}{1-\rho}} d_{k}}$ Como $\int_{0}^{m} P_{j} C_{j} d_{j} \equiv \int_{0}^{m} P_{k} C_{k} d_{k} \equiv E$, se tiene que

$$
C_{j}\left(P_{j}, P_{-j}, E\right)=\frac{E P_{j}{ }^{-\frac{1}{1-\rho}}}{\int_{0}^{m} P_{k}{ }^{-\frac{\rho}{1-\rho}} d_{k}} ;
$$

corresponde a la función de demanda marshalliana del bien $j$ en función de los precios de todas las variedades y del gasto monetario total en ellas. Si $m$ es grande (alta variedad de páginas web), un cambio en $p_{j}$ no tiene 
un efecto sobre $E$ y sobre $\int_{0}^{m} P_{k}^{-\frac{\rho}{1-\rho}}$, por ende $-\frac{\partial c_{j}}{\partial P_{j}} \frac{P_{j}}{C_{j}}=\frac{1}{1-\rho}=\theta$, de manera que $\theta$ también corresponde a la elasticidad precio de demanda de la variedad $c_{j}$ con respecto a su precio. En [9], $\left[\int_{0}^{m} P_{k}^{-\frac{\rho}{1-\rho}} d_{k}\right]^{-\frac{(1-\rho)}{\rho}}$ corresponde al índice de precios dual a Z, de modo que [9] puede expresarse como

$$
\begin{gathered}
C_{j}=\frac{E P_{j}^{-\frac{1}{1-\rho}}\left[\int_{0}^{m} P_{k}^{-\frac{\rho}{1-\rho}} d_{k}\right]^{-\frac{1}{\rho}}}{\left(\int_{0}^{m} P_{k}^{-\frac{\rho}{1-\rho}} d_{k}\right)\left(\int_{0}^{m} P_{k}^{-\frac{\rho}{1-\rho}} d_{k}\right)^{-\frac{1}{\rho}}} \\
=\frac{E P_{j}^{-\frac{1}{1-\rho}}}{P_{d}\left(\int_{0}^{m} P_{k}^{-\frac{\rho}{1-\rho}} d_{k}\right)^{\frac{1}{\rho}}} \\
\text { Como } E=Z P_{d}, \text { ya que } \int_{0}^{m} P_{j} C_{j} d_{j}= \\
\left(\int_{0}^{m} C_{j}^{p}\right)^{1 / \rho}\left(\int_{0}^{m} P_{j}^{-\frac{\rho}{1-\rho}} d_{j}\right)^{1 / \rho}, \text { la expresión } \\
\text { anterior equivale a } C_{j}=\frac{Z\left[\left(\int_{0}^{m} P_{k}^{-\frac{\rho}{1-\rho}} d_{k}\right)\right.}{\left.-\frac{(1-\rho)}{\rho}\right]^{-\frac{1}{1-\rho}}} \\
\text { así que } c_{j}=Z\left(\frac{P_{d}}{P_{j}}\right)^{\frac{1}{1-\rho}} \text { a } P_{j}^{\frac{1}{1-\rho}}
\end{gathered}
$$

Sea $q_{j}$ la oferta agregada de la variedad disponible en la página web $j$. Esta es producida por un administrador-vendedor $j$ en condiciones de competencia monopolística. En equilibrio de mercado, esta debe ser igual a su demanda individual multiplicada por el número total de consumidores, de manera que se clarifique este sub-mercado específico. La ecuación [10] establece esta condición:

$$
q_{j}=n C_{j} .
$$

En consecuencia, la demanda agregada de $\mathrm{j}$ es

$$
q_{j}=n Z\left(\frac{P_{d}}{P_{j}}\right)^{\frac{1}{1-\rho}}
$$

Dado que la elasticidad de sustitución es mayor a uno, puede probarse que se trata de una función cóncava decreciente en $p_{j}$. La función inversa de demanda para el bien $j$ :

$P_{j}=P_{d}\left(\frac{n Z}{q_{j}}\right)^{1-\rho}$

La Figura 2 muestra gráficamente la curva inversa de demanda de la variedad-página web $j$.

Figura 2. Dinámica del comportamiento de los consumidores en el criptomercado de drogas ilegales

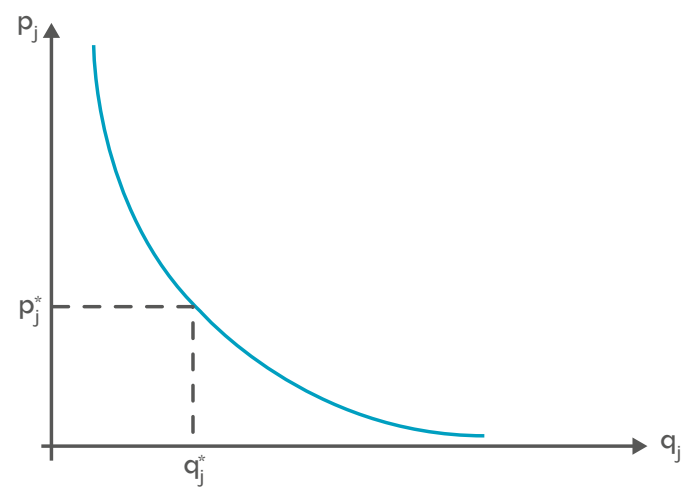

Fuente: elaboración propia.

\section{b. Segunda etapa:}

Dada la dinámica de la estructura de mercados de competencia monopolística, el análisis se plantea a corto y largo plazo. Adicionalmente, se define una variable en la función de ganancias del traficante, que expresa la probabilidad de que un porcentaje de drogas transadas en una página sea rastreado y decomisado. Dicha captura/decomiso puede implicar el cierre de las páginas web, viéndose comprometidas las entregas de las ventas ya realizadas.

Este efecto es importante, porque las bandas criminales que transan en el criptomercado de drogas ilegales reducen su nivel de exposición física en el momento transaccional, lo cual disminuye la posibilidad de captura de la droga y del traficante. En el criptomercado, el principal riesgo se expresa como un costo de oportunidad, dado que la potencial vulnerabilidad frente a las autoridades de control se centra en el 
cierre de la página web y en menor grado, el decomiso de la droga.

Por ser una actividad ilegal, en el momento de la entrega del producto se puede presentar una captura de las drogas; por ende, es obligatorio introducir una variable de confiscación $(\delta)$. Esta probabilidad de captura/decomiso de las drogas ilegales se considera exógena en este modelo. Cabe agregar que debido a que los costos son simétricos entre todas las firmas, y la demanda que todas enfrentan es idéntica en términos cuantitativos (a pesar de la existencia de diferenciación horizontal), existe simetría en los problemas a resolver por parte de todas las firmas, así como en los resultados obtenidos. Según lo anterior, las ganancias $\left(\pi_{j}\right)$ del administrador-vendedor $j$ se deben interpretar como una función de ganancias esperadas.

\section{b.1 Corto plazo:}

En el corto plazo, el problema de la firma vendedora en el criptomercado $j$ es:

$$
\operatorname{Max}_{q_{j}} \pi_{j}=P_{j}\left(q_{j}\right) q_{j}(1-\delta)-B_{j}\left(q_{j}\right)
$$

Al sustituir [3] en [1 1] se obtiene

$$
\operatorname{Max}_{q_{j}} \pi_{j}=P_{j}\left(q_{j}\right) q_{j}(1-\delta)-b q_{j}-F
$$

La C.P.O. es:

$$
\begin{gathered}
\frac{\partial \pi_{j}}{\partial q_{j}}=o ;\left[P_{j}^{\prime}\left(q_{j}\right) q_{j}+P_{j}\left(q_{j}\right)\right](1-\delta)-b=0[12] \\
{\left[P_{j}^{\prime}\left(q_{j}\right) q_{j}+P_{j}\left(q_{j}\right)\right](1-\delta)=b .}
\end{gathered}
$$

Esta condición establece que, de acuerdo con la política de precios, el ingreso marginal de cada firma vendedora es igual a su costo marginal de producción.

$$
P_{j}(1-\delta)\left[1+\frac{P_{j}^{\prime}\left(q_{j}\right) q_{j}}{P_{j}}\right]=b
$$

Aquí $-\frac{P^{\prime}{ }_{j}\left(q_{j}\right) q_{j}}{P_{j}}$ corresponde al inverso de la elasticidad precio de demanda, de modo que la expresión anterior se puede expresar como:

$$
P_{j} \rho(1-\delta)=b
$$

Esta ecuación define la política de precios óptima de la firma monopolística en el corto plazo, bajo la conjetura de ganancias esperadas, dada la probabilidad de captura de una transacción $(\delta)$, producto de la actividad ilegal.

Se observa que la estructura de precios es del tipo $P_{j}=(1+\mu) b$, donde $\mu$ expresa el margen de ganancias constantes de los traficantes. De [12'] tenemos que: $P_{j}=\frac{b}{\rho(1-\delta)}$, por lo tanto, $(1+\mu) b=\frac{b}{\rho(1-\delta)}$, y se obtiene un markup constante e igual a:

$$
\mu=\frac{1-\rho(1-\delta)}{\rho(1-\delta)}
$$

Por último, el ingreso total de la firma $j$ es $\tilde{I}_{j}\left(q_{j}\right)=P_{j}\left(q_{j}\right) q_{j}(1-\delta)$; reemplazando la función inversa de demanda $\left[P_{j}\left(q_{j}\right)\right]$ en $\tilde{I}_{j}\left(q_{j}\right)$, se obtiene:

$$
\tilde{\mathrm{I}}_{j}\left(q_{j}\right)=p_{d}(n Z)^{1-\rho} q_{j}{ }^{\rho}(1-\delta)
$$

Entonces,

$$
\frac{\partial \tilde{\mathrm{I}}_{j}}{\partial q_{j}}=\rho q_{j}^{(\rho-1)} p_{d}(n Z)^{1-\rho}(1-\delta)>0,
$$

$\frac{\partial^{2} \tilde{\mathrm{I}}_{j}}{\partial q_{j}{ }^{2}}=\rho(\rho-1) q_{j}^{(\rho-2)} p_{d}(n Z)^{1-\rho}(1-\delta)<0$

De manera que la función de ingreso total es creciente y cóncava para todo nivel de $q_{j}$.

Bajo la estructura de mercado de competencia monopolística, en el corto plazo el precio siempre es mayor que el costo marginal, $P_{j}>b$. No obstante, en el corto plazo las ganancias de los vendedores online pueden ser positivas, iguales a cero, o negativas, dependiendo de si el precio es mayor, igual o menor que el costo medio de producción, respectivamente. Por lo tanto, si el precio fuera mayor al costo medio de producción, $\pi_{j}^{c p}>0$, existirían incentivos para la entrada de nuevos traficantes al criptomercado de drogas ilegales, con otras varieda- 
des en nuevas páginas web; lo contrario ocurriría si sus ganancias de corto plazo fueran negativas.

Dado que los ingresos del traficante están inversamente relacionados con la probabilidad de captura de una transacción $(\delta)$, la represión de las autoridades de control frente a dichas actividades puede verse como efectiva en el objetivo de reducir dichas transacciones ilegales. Sin embargo, en la primera etapa del juego se podrá comprobar que, debido a la adaptabilidad estratégica de los agentes criminales y a la rapidez de las externalidades en red de la demanda, estos pueden contrarrestar el objetivo mismo de la represión que en el presente modelo está captado por el parámetro $\delta$.

De las expresiones anteriores, se obtiene el equilibrio de corto plazo que se muestra en la Figura 3.

Figura 3. Estructura de ingresos y producción de los traficantes en el corto plazo

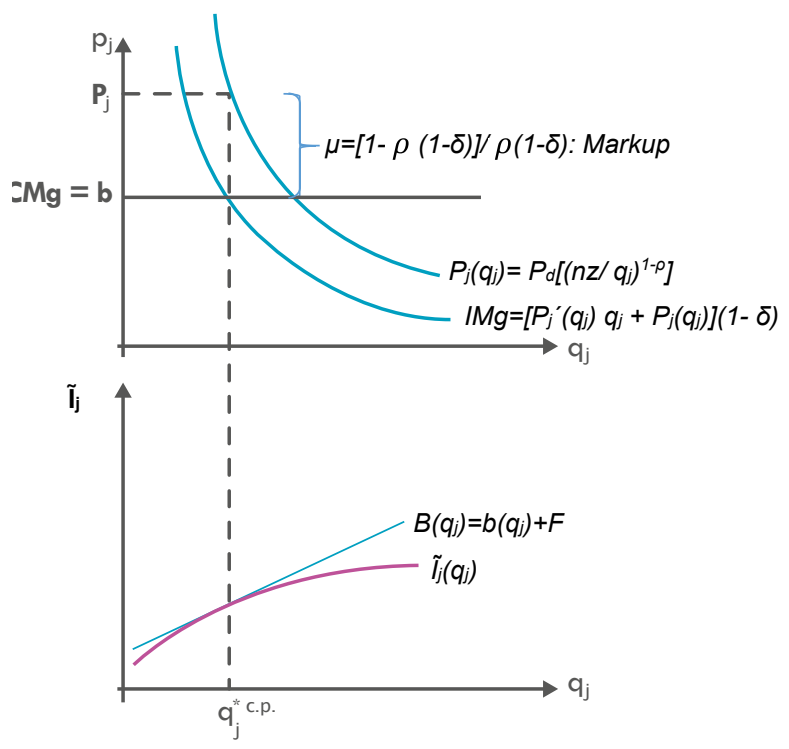

Fuente: elaboración propia.

\section{b. 2 Largo plazo:}

La condición de largo plazo para las firmas traficantes exige que las ganancias sean cero en el largo plazo:

$$
\pi_{j}=P_{j}\left(q_{j}\right) q_{j}(1-\delta)-b q_{j}-\mathrm{F}=0 ;
$$

Por lo tanto,

$$
\begin{gathered}
P_{j}\left(q_{j}\right) q_{j}(1-\delta)=b q_{j}+\mathrm{F} \\
P_{j}=\frac{b q_{j}+\mathrm{F}}{q_{j}(1-\delta)} \\
P_{j}=\frac{1}{(1-\delta)}\left[b+\frac{\mathrm{F}}{q_{j}}\right],
\end{gathered}
$$

ecuación que muestra que el precio es igual al costo medio de producción en el equilibrio de largo plazo.

Utilizando la condición de corto plazo, se obtienen las ventas de equilibrio para el vendedor $j$. Como se trata de un equilibrio simétrico, se tiene que:

$$
q *=\left(\frac{\rho}{1-\rho}\right) \frac{\mathrm{F}}{b}
$$

Con [10] se obtiene el nivel de consumo de equilibrio de largo plazo:

$$
C^{*}=\left(\frac{\rho}{1-\rho}\right) \frac{\mathrm{F}}{b n}
$$

Los precios de equilibrio de largo plazo son iguales a los de corto plazo, de manera que también están definidos por la ecuación [12'], despejando $P_{j}$ :

$$
P_{j}=\frac{b}{\rho(1-\delta)}
$$

Esto se debe a que la elasticidad precio de demanda es constante, de modo que el equilibrio de largo plazo produce un ajuste de cantidades, mas no de precios, con respecto al de corto plazo.

Dado que la condición de largo plazo se sostiene para cada traficante en el criptomercado, los ingresos totales de dicho mercado deben igualarse a los costos fijos totales. Además, los ingresos totales deben igualarse a los gas- 
tos totales de los consumidores, quienes gastan una combinación de variedades-páginas web, de modo que

$$
\mathrm{mF}=n E
$$

Por lo tanto, el equilibrio de largo plazo se puede visualizar en la Figura 4.

Figura 4. Estructura de ingresos y producción de los traficantes en el largo plazo

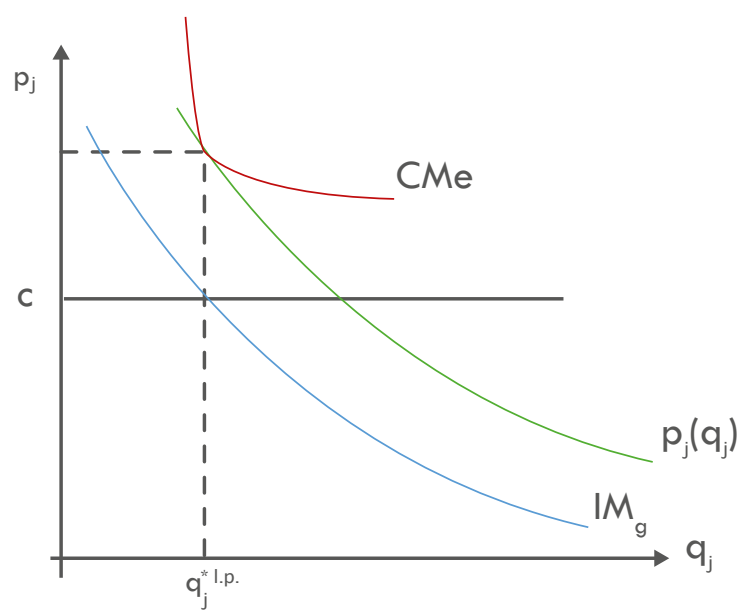

Fuente: elaboración propia.

\section{c. Primera etapa:}

Los consumidores eligen el nivel de gasto total en variedades de drogas-página web, $E$, y con esto deciden si adquieren los dispositivos técnicos básicos para acceder al criptomercado, conociendo los principales resultados de las etapas posteriores del juego (funciones de reacción de los vendedores en la segunda etapa y de ellos mismos en la tercera etapa). Despejando $C_{0}$ de [2], se obtiene

$$
C_{0}=\hat{\mathrm{I}}-P_{B}-E
$$

Reemplazando esta expresión en la función de utilidad, se obtiene

$$
\mathcal{U}=\hat{\mathrm{I}}-P_{B}-E_{0}+\phi\left[\left(\int_{0}^{m} C_{j}^{\rho} d_{j}\right)^{1 / \rho}\right]^{\beta} .
$$

De [16] se tiene que

$$
\mathrm{m}=\frac{n E}{\mathrm{~F}}
$$

Esto permite endogenizar el número óptimo de drogas-página web, en función del número de consumidores, del gasto total en las variedades-páginas web y de los costos fijos. Dada la simetría del equilibrio y con la condición de clarificación de los mercados online, la utilidad en equilibrio viene dada por

$\mathcal{U}=\hat{\mathrm{I}}-P_{B}-E+\phi\left[\left(\int_{0}^{m}\left(\frac{q}{n}\right)^{p} d_{j}\right)^{1 / \rho}\right]^{\beta}$

Dado que de [10] $q=n C \Rightarrow C=\frac{q}{n}$, y por la simetría en el consumo de variedades se llega a

$$
\mathcal{U}=\hat{\mathrm{I}}-P_{B}-E+\phi\left(\frac{q}{n}\right)^{\beta} m^{\beta / \rho}
$$

Con [16], se obtiene

$$
\mathcal{U}=\hat{\mathrm{I}}-P_{B}-E+\left(\phi \frac{q^{\beta} E^{\alpha}}{F^{\alpha}}\right) n^{\beta(1-\rho) / \rho}
$$

Donde $\alpha=\frac{\beta}{\rho}$. Definiendo $\tau \equiv \frac{q^{\beta}}{F^{\alpha}}$ y se obtiene

$$
\mathcal{U}=\hat{\mathrm{I}}-P_{B}-E+\phi \tau n^{\frac{\alpha}{\theta}} E^{\alpha}
$$

Ya que $\frac{\beta}{\rho}(1-\rho)=\frac{\alpha}{1-\rho}=\frac{\alpha}{\theta}$.

La ecuación [17] es una expresión de la función de utilidad indirecta, dado que los consumidores conocen las funciones de reacción de los vendedores ilegales y de ellos mismos en las etapas posteriores del juego. Como era de esperarse, la función indirecta de utilidad depende del ingreso de los consumidores, del precio de los elementos técnicos básicos para acceder al criptomercado y a la combinación drogas-página web, $P_{B}$, de su gasto de equilibrio en las variedades de drogas-páginas web, $E$, pero también del número total de consumidores, $n$. Como veremos en mayor detalle en lo que sigue, la influencia de $n$ en la utilidad indirecta capta los efectos de externalidades en red que tiene la presencia de un número 
determinado de consumidores en el criptomercado de drogas online.

Así, el problema de los consumidores en la primera etapa para determinar el nivel de gasto agregado en $E^{*}$ es

$$
\operatorname{Max}_{\{E\}} \hat{\mathrm{I}}-P_{B}-E+\phi \tau n^{\frac{\alpha}{\theta}} E^{\alpha}
$$

La C.P.O. es

$$
\frac{\partial u}{\partial E}=0 ;-1+\alpha \phi \tau n^{\frac{\alpha}{\theta}} E^{\alpha-1}=0
$$

De donde se puede hallar el gasto agregado en variedades de equilibrio, $E^{*}$ :

$$
E^{*}=\left(\alpha \phi \tau n^{\frac{\alpha}{\theta}}\right)^{\frac{1}{1-\alpha}}
$$

La expresión [19] define el equilibrio en el gasto del consumidor; lo interesante del resultado es que dicho gasto depende del número de consumidores de la variedad droga-página web, evidenciando de nuevo un claro proceso de externalidad en red en el gasto de dicho bien.

Ahora, de [19] en [17] se obtiene

$\mathcal{U}=\hat{\mathrm{I}}-P_{B}-\left(\left(\alpha \phi \tau n^{\frac{\alpha}{\theta}}\right)^{\frac{1}{1-\alpha}}\right)$

$+\phi \tau n^{\frac{\alpha}{\theta}}\left(\alpha \phi \tau n^{\frac{\alpha}{\theta}}\right)^{\frac{\alpha}{1-\alpha}}$

Con un poco de álgebra se obtiene

$\mathcal{U}=\hat{\mathrm{I}}-P_{B}+\left(\frac{1-\alpha}{\alpha}\right) \phi^{\frac{1}{1-\alpha}}(\alpha \tau)^{\frac{1}{1-\alpha}} n^{\frac{\alpha}{\theta(1-\alpha)}}$

Si se define que $\kappa=\left(\frac{1-\alpha}{\alpha}\right)(\alpha \tau)^{\frac{1}{1-\alpha}}$, se obtiene

$$
\mathcal{U}=\hat{\mathrm{I}}-P_{B}+\phi^{\frac{1}{1-\alpha}} \kappa n^{\frac{\alpha}{\theta(1-\alpha)}},
$$

la cual constituye una expresión reducida de la utilidad indirecta. Para obtener una expresión más clara de la utilidad del consumidor, se define la siguiente relación:

$$
H(n) \equiv \kappa n^{\frac{\alpha}{\theta(1-\alpha)}}
$$

En general, $H(n)$ se puede entender como una transformación monotónica de $n$. Por ende, en términos generales la función de utilidad indirecta del consumidor se puede expresar como:

$$
\mathcal{U}=\hat{\mathrm{I}}+\omega H(n)-P_{B}
$$

Donde $\omega \equiv \phi^{\frac{1}{1-\alpha}}$, y

$$
\frac{\partial H}{\partial \mathrm{n}}=\frac{\alpha}{\theta(1-\alpha)} \kappa n^{\frac{\alpha}{\theta(1-\alpha)}-1}>0
$$

Con esto se comprueba que los efectos indirectos de red pueden surgir en un contexto de mercado, por el efecto de la participación del consumidor (que depende de su intensidad en el uso de las páginas web, de la reputación de los vendedores online). De [19] se obtiene una de las conclusiones fundamentales del modelo: en su forma reducida, la utilidad indirecta del consumidor depende del número de consumidores, lo cual cobra mayor sentido al tener en cuenta que el nivel de reputación refuerza la retención o incrementa el número de consumidores que transan la variedad drogas-página web, lo que a su vez propicia la aparición de nuevas páginas web generando nuevas variedades. La siguiente proposición formaliza este resultado:

Proposición 2: En equilibrio, la función de utilidad indirecta es una función creciente cónca$v a$, lineal o convexa del número de consumidores presentes en el criptomercado.

Prueba: Obsérvese que $H(n)=\kappa n^{\frac{\alpha}{\theta(1-\alpha)}}$, por lo tanto,

$$
\frac{\partial H(n)}{\partial n}=\frac{\alpha}{\theta(1-\alpha)} \kappa n^{\frac{\alpha}{\theta(1-\alpha)}-1}>0,
$$

dado que

$0<\alpha=\frac{\beta}{\rho}<1 \Rightarrow 0<(1-\alpha)=\frac{\rho-\beta}{\rho}<1$.

Adicionalmente, se tiene que

$\frac{\partial^{2} H(n)}{\partial n^{2}}=\frac{\alpha}{\theta(1-\alpha)}\left(\frac{\alpha}{\theta(1-\alpha)}-1\right) \kappa n^{\frac{\alpha}{\theta(1-\alpha)}-2}$.

Dado que $\frac{\alpha}{\theta(1-\alpha)}=\frac{\frac{\beta}{\rho}}{\left(\frac{1}{1-\rho}\right)\left(\frac{\rho-\beta}{\rho}\right)}=\frac{(1-\rho) \beta}{(\rho-\beta)}$

$y \frac{\alpha}{\theta(1-\alpha)}-1=\frac{\beta(2-\rho)-\rho}{(\rho-\beta)} \gtreqless 0$. 
Si $y$ solo si $\beta \gtreqless \frac{\rho}{2-\rho}$. Entonces se tiene que

$$
\begin{aligned}
& \text { i) } \quad \beta>\frac{\rho}{2-\rho} \Rightarrow \frac{\partial^{2} H(n)}{\partial n^{2}}>0 \\
& \text { ii) } \quad \beta=\frac{\rho}{2-\rho} \Rightarrow \frac{\partial^{2} H(n)}{\partial n^{2}}=0 \\
& \text { iii) } \quad \beta<\frac{\rho}{2-\rho} \Rightarrow \frac{\partial^{2} H(n)}{\partial n^{2}}<0
\end{aligned}
$$

Por la condición de estabilidad de la función de utilidad del consumidor -ver ecuación [1]-, en cualquier caso debe tenerse que $\beta<\rho$. Lo que efectivamente se cumple en los tres casos posibles puesto que $0<\rho<1, y \rho<\frac{\rho}{2-\rho}$

En última instancia, la elección de comprar o no los elementos técnicos básicos para acceder a la web oscura y, a su vez, acceder y transar en el criptomercado, se define de la siguiente manera: compra los elementos técnicos básicos ${ }^{9} \mathrm{si}$

$$
\hat{\mathrm{I}}+\omega H(n) \geq P_{B}
$$

No compra los elementos técnicos básicos si

$$
\hat{\mathrm{I}}+\omega H(n)<P_{B}
$$

$H(n)$ no es más que una función de externalidades, la cual, según el resultado obtenido, refuerza el papel del efecto colateral positivo de un consumidor de drogas-página web en la elección de este bien por parte de otros consumidores, lo cual se evidencia explícitamente en el nivel de reputación de las páginas web del criptomercado de drogas ilegales.

Como la Proposición $1 H(n)$ es una función creciente en $n$, puede definirse un nivel umbral de $n$ a partir del cual los consumidores acceden a los dispositivos técnicos necesarios para comprar drogas en la web oscura. La Definición 1 establece esto formalmente:

9 En concreto, los consumidores hacen uso de un encriptador de rutas (TOR-VPN) y un encriptador de mensajes (PGP).
Definición 1: Sea ñ el número de consumidores online más allá del cual estos adquieren los dispositivos técnicos necesarios para comprar drogas en la web oscura. De [22], teniendo en cuenta que $H(n)=\kappa n^{\frac{\alpha}{\theta(1-\alpha)}}$, este nivel corresponde $a$ $\tilde{n}=\left(\frac{P_{B}}{\omega k}\right)^{\frac{\theta(1-\alpha)}{\alpha}}$

Expresado en términos de los parámetros primitivos y precios del modelo, este nivel de umbral corresponde a

$$
\tilde{n}=\left[\frac{\left(P_{B}\left(\frac{\beta}{\rho-\beta}\right)\right)^{\frac{\beta}{\rho-\beta}}}{\phi \frac{\beta}{\rho}\left(P_{j}^{*}\right)^{\beta}(F)^{-\left(\frac{1}{\rho}\right)}}\right]^{\theta} .
$$

Esta expresión muestra que $\tilde{n}$ depende positivamente de los costos fijos de los vendedores, $F$, y del precio de los dispositivos para acceder a la red oscura, $P_{B}$. También depende negativamente del precio de equilibrio de cada variedad de droga-página web, $P_{j}^{*}$, y del parámetro $\phi$.

Este nivel umbral de consumidores implica que el propio funcionamiento del criptomercado de drogas es viable solo cuando el número de consumidores supera una escala mínima, a partir de la cual estos efectivamente adquieren los dispositivos necesarios para acceder a la web oscura.

\section{Principales hallazgos}

\section{a. Tercera etapa:}

La Tabla 1 permite detallar los resultados de estática comparativa respecto a la función inversa de demanda, los cuales sintetizan los resultados del juego en la tercera etapa.

Como era de esperarse, un incremento en el índice de precios de la industria, $p_{d}$, da lugar a un aumento en el precio de la variedad $j$, debido a que lleva a descensos en la demanda de todas las variedades-página web sustitutas y a incrementos en su nivel de demanda. La Figu- 
Sociedad y Economía N 44 (Sep - dic 2021) / e-ISSN: 2389-9050 / e10610962

Leonardo Raffo, Diego Gómez-Calderón y Carolina Ramírez-Andrade

Tabla 1. Estática comparativa para la función inversa de demanda

\begin{tabular}{|c|c|c|}
\hline $\begin{array}{c}\text { Condición de } \\
\text { cambio }\end{array}$ & Resultado & Relación \\
\hline$\frac{\partial p_{j}}{\partial p_{d}}$ & $\left(\frac{n Z}{q_{j}}\right)^{1-\rho}>0$ & Positiva \\
\hline$\frac{\partial p_{j}}{\partial \mathrm{n}}$ & $(1-\rho) \frac{p_{d}}{n}\left(\frac{n Z}{q_{j}}\right)^{1-\rho}>0$ & $\begin{array}{c}\text { Positiva } \\
\text { (1- } p_{j}\end{array}$ \\
\hline$\frac{\partial p_{j}}{\partial \mathrm{Z}}$ & $(1-\rho) \frac{p_{d}}{Z}\left(\frac{n Z}{q_{j}}\right)^{1-\rho}>0$ & Positiva \\
\hline$\frac{\partial p_{j}}{\partial q_{j}}$ & $-(1-\rho) \frac{p_{d}}{q_{j}}\left(\frac{n Z}{q_{j}}\right)^{1-\rho}<0$ & Negativa \\
\hline
\end{tabular}

Fuente: elaboración propia en 2020.

ra 1 muestra gráficamente la curva inversa de demanda de la variedad-página web $j$.

De igual forma, $\frac{\partial p_{j}}{\partial n}, \frac{\partial p_{j}}{\partial z} y$ expresan que incre-

mentos (disminuciones), en el tamaño del mercado y en el bien compuesto $Z$ (con todas las variedades existentes), generan incrementos (descensos) en $p_{j}$. Este efecto genera mayores ganancias en el corto plazo para los traficantes, ya que una nueva variedad $j$ enfrenta una mayor demanda; pero, como consecuencia, se generan incentivos para la entrada de nuevos traficantes y, con ello, de nuevas variedades de droga-página web, en el largo plazo, eliminando dicho poder de mercado. El impacto positivo de $Z$, tanto sobre $p_{j}$ como sobre $q_{j}$ en la función de demanda agregada -ver ecuación [8'"]-, constituye una externalidad positiva del número de variedades de la industria sobre la demanda de una variedad específica.

\section{b. Segunda etapa:}

La competencia en los criptomercados establece diferenciadores desde la estructura tecnológica, lo que conduce a una saturación de oferta en el criptomercado y lleva a que los consumi- dores se concentren solo en algunas de ellas. Esto ratifica el comportamiento del modelo de competencia monopolística. En el largo plazo, se genera una estabilización del mercado con aquellas firmas capaces de competir bajo las condiciones de mercado.

Lo anterior implica que la regulación de los mercados de drogas ilegales se da tanto por los mecanismos propios de mercado como por la represión de las autoridades de control, ya que la probabilidad de captura de una transacción $\delta$ afecta negativamente las ganancias de la firma. Un incremento en $\delta$ produce una contracción de la oferta y un incremento de los precios en el corto plazo, pero paradójicamente genera nuevamente incentivos para la aparición de nuevas variedades tecnológicas debido a la competencia bajo libre entrada y salida de firmas. El resultado es que en el largo plazo, si bien los precios se mantienen en un nivel más alto -debido a que los precios de equilibrio de corto plazo coinciden con los de largo plazo-, la oferta de drogas vuelve a su nivel inicial. Esto configura un claro proceso de adaptabilidad estratégica de los agentes criminales, que revierte el efecto inicial de la política criminal. 
De la ecuación [14] puede corroborarse directamente que el impacto neto de la política criminal $(\delta)$ en el nivel transaccional de cada variedad a largo plazo es nulo, puesto que $q^{*}$ no depende en el largo plazo de $\delta$. La siguiente proposición formaliza este resultado.

Proposición 3: En equilibrio, un incremento en la probabilidad de interdicción produce un incremento en los precios de equilibrio, y de ahí en las ganancias de corto plazo. No obstante, en el largo plazo entran nuevos traficantes al criptomercado, dando como resultado ganancias nulas y un efecto nulo sobre las ventas de equilibrio.

Prueba: Directamente de la ecuación [12], despejando el precio de equilibrio (el de corto plazo es igual al de largo plazo), y de la ecuación [14] $\mathbf{\square}$.

Asimismo -de la ecuación [15]-, puede verse que los niveles de consumo de equilibrio de cada variedad tampoco dependen directamente de $\delta$.

Lo que explica estos resultados en la realidad es que un fortalecimiento de la política de interdicción por parte de las autoridades de control, expresado por un incremento en la probabilidad de detección y arresto, lleva a una reducción de las transacciones ilegales, vía cierre de páginas web en el criptomercado de drogas ilícitas tan solo en el corto plazo. En el largo plazo los administradores de las páginas web reprimidas tienden a eludir a las autoridades de control con mayor facilidad que en un mercado tradicional de drogas ilícitas (territorio físico), abriendo nuevas páginas web. Adicionalmente, la competencia en un contexto con mayores precios y mayores ganancias en el corto plazo, produce incentivos muy fuertes para la entrada de nuevos vendedores a la red oscura. Lo anterior devela un claro proceso de adaptabilidad estratégica criminal que no era viable en el caso de los mercados de drogas tradicionales.

El principal efecto de la política criminal es su relación directa con el precio, evidenciando que a mayor probabilidad de detección y arresto se define un mayor precio por variedad, y viceversa; lo cual se explica por el mayor ries- go transaccional. Esta es una de las principales justificaciones de la represión frente a los mercados de drogas ilícitas; sin embargo, este mecanismo de represión genera una dinámica de adaptabilidad estratégica en los traficantes que, en el caso del criptomercado de drogas ilícitas, se evidencia por la sofisticación tecnológica del espacio transaccional. Lo anterior reduce o invalida el efecto represivo frente al precio en el largo plazo, al generarse incentivos para una nueva entrada de firmas y una consecuente apertura de nuevas páginas web.

El análisis derivado de la probabilidad de captura y detección $\delta$ indica el enorme desafío de la política criminal frente al criptomercado, ya que la adaptabilidad estratégica en un espacio transaccional siempre potencialmente creciente es mayor a la maniobrabilidad que tienen los criminales en el espacio físico (territorial).

\section{c. Primera etapa:}

En la primera etapa del modelo se validan las externalidades en red que se generan en las dinámicas de los criptomercados de drogas ilegales. En estos procesos virtuales, las externalidades están relacionadas con el nivel reputacional de las páginas web, que permite retener o incrementar el número de consumidores de drogas ilegales y, a su vez, propiciar la aparición de nuevas páginas web con nuevas variedades tecnológicas.

Adicionalmente, se demuestra que, en su forma reducida, la utilidad del consumidor depende del número de consumidores en la web oscura y en las páginas web. $H(n)$ permite evidenciar el efecto colateral positivo de un consumidor de drogas-página web en la elección de este bien por parte de otros consumidores, por cuenta del nivel de reputación de las páginas web del criptomercado de drogas ilegales.

A diferencia del mercado convencional de drogas ilegales, en los criptomercados la velocidad de la transmisión del nivel reputacional de una página web es mayor, permitiendo que los consumidores generen tendencias de moda en el consumo de droga, por ejemplo. 
De otra parte, con las ecuaciones [14] y [15] queda claro que, como $q^{*}$ y $C^{*}$ no dependen de la probabilidad de interdicción, el único efecto de la política de interdicción sobre la utilidad indirecta de equilibrio se da de forma indirecta a través del efecto de externalidades en red de los consumidores online: al incrementarse la probabilidad de captura, $\delta$, se incrementa en el largo plazo el número de vendedores ilegales online y, como consecuencia, $E^{*}$-de ecuación [19]- y el número de consumidores que efectivamente acceden a los dispositivos necesarios para acceder a las páginas web ilegales. Esto último debido a que el número de consumidores para los que $\tilde{I}+\omega H(n) \geq P_{B}$ crece, de manera que el número de consumidores que efectivamente acceden a comprar drogas en la red oscura aumenta. El resultado de todo esto es un efecto de externalidades en red mayor sobre la utilidad indirecta.

Este efecto contraproducente de la política de interdicción implica la existencia de mecanismo de feedback (retroalimentación) entre $n$ y $m$, que conlleva a la potenciación de los efectos de externalidad en red de $n$ : un mayor nivel de $n$ por factores exógenos lleva a incrementos en el número de vendedores $m$, tal como lo predice el modelo canónico de Dixit y Stiglitz (1977). Por otra parte, un incremento en $m$ producido por un endurecimiento de la política de interdicción -un mayor nivel de $\delta$, induce a la entrada de un mayor número de consumidores efectivos al criptomercado. Este efecto constituye un mecanismo adicional de retroacción positiva que no es capturado por el modelo tradicional de Dixit y Stiglitz (1977).

Lo anterior genera importantes desafíos para las autoridades de control, ya que la afectación a la oferta vía represión genera un impacto indirecto positivo sobre el nivel de utilidad de los consumidores, los cuales vía externalidades en red impulsan el surgimiento de nuevas firmas (páginas web) en el mercado. Así, el efecto de las externalidades en red en la aparición de nuevas firmas se potencia en el espacio del criptomercado de drogas ilegales, paradójicamente, al endurecerse la política de represión a los criptomercados.

\section{Política antidrogas frente a la dinámica del criptomercado de drogas ilegales}

El criptomercado de drogas ilegales representa un modelo de negocio que requiere conocimiento especializado y destreza técnica, bien sea para acceder a él a través de la web oscura, o para desarrollar las diferentes páginas web y comercializar las drogas ilícitas. Los traficantes se comportan de forma estratégica, lo cual hace que puedan evadir constantemente a las autoridades de control.

En este sentido, las autoridades de control también deben desarrollar una adaptabilidad estratégica frente a este nuevo modelo de negocio de los traficantes. Dicha adaptabilidad implica no solo adquirir herramientas tecnológicas para la detección de operaciones ilegales en la web oscura, sino también entender la dinámica del criptomercado y la versatilidad de los traficantes para invisibilizar sus transacciones.

Aquí el punto clave que revela el modelo es que las autoridades no solamente deben detectar y cerrar páginas web encriptadas de forma eficiente, sino al mismo tiempo desarrollar los mecanismos informáticos que impidan a los vendedores migrar a otras páginas web para continuar con su accionar delictivo. Esto implica identificar rápidamente las posibles rutas de migración, pero también desarrollar algoritmos de bloqueo en tiempo mínimo de estas.

Un reto adicional para las autoridades de control será mejorar sus actividades de inteligencia sin violar los derechos de los ciudadanos al libre manejo de su información. En diferentes países, hay antecedentes sobre las exigencias ciudadanas al Estado para hacer uso libre de la Internet y, además, mantener privada la información sobre su navegación en línea.

Las acciones de coerción por parte de las autoridades de control en los mercados tradicionales incentivan a los traficantes a comercializar drogas ilegales en páginas web para usufruc- 
tuarse de estas herramientas tecnológicas, mientras invisibilizan su operación. Lo anterior puede generar percepciones erradas sobre la efectividad de los mecanismos de coerción, toda vez que los traficantes no desaparecen, sino que migran a un espacio web en el que los eslabones de comercialización y distribución de drogas están menos expuestos que en los mercados tradicionales. Cabe recordar que el uso de páginas web de drogas ilegales incrementa las ganancias para los traficantes debido a la reducción de intermediarios en la transacción ilegal.

En países como Colombia, la cadena de producción, tráfico y distribución de las drogas ilegales involucra personas de diversa condición socioeconómica (De-León y Salcedo, 2014; Duncan, 2014). Sin embargo, en el caso de los distribuidores al por menor (los jíbaros en el argot popular) son personas con escasos niveles de capital humano quienes laboran. No obstante, debido a las exigencias en el manejo de software e ingeniería de sistemas, en el caso de los criptomercados la distribución de las drogas al por menor -y también al por mayor, en el caso de las páginas web que también transan drogas al por mayor- exige altos niveles de capital humano y experticia para el acceso y navegación en la red profunda (Kruithof et al. 2016; García-Sigman, 2017). Esto plantea nuevos retos a las autoridades de control, ya que se trata de un delito cuya ejecución despliega un nivel muchísimo mayor de inteligencia logística.

Adicionalmente, esta sofisticación de los procesos criminales en los cuales se necesitan personas con conocimientos técnicos especializados genera incentivos perversos para que una fracción de la población con altos niveles de capital humano -en presencia de altas tasas de desempleo- se movilice hacia actividades criminales.

Si bien la dinámica de los criptomercados se presenta principalmente en los países desarrollados -en los cuales sus ciudadanos cuentan con mayor acceso a herramientas tecnológicas, capacidades frente al funcionamiento de las plataformas ilegales e ingresos-, en Colombia ya hay registros del uso de herramientas tecnológicas para la comercialización de drogas ilegales, como WhatsApp, por ejemplo (El Tiempo, 2018).

Aunque el artículo se centra en las transacciones en el criptomercado de drogas ilegales y las páginas que estos contienen, se debe tener en cuenta que los criptomercados ilegales son espacios de mercado en los que se pueden comercializar otro tipo de bienes, por lo que también existen otros criptomercados: de armas, libros, documentos falsos, medicamentos, software, entre otros (ver Martin, 2014; Dolliver, 2015; Hardy y Norgaard, 2015; entre muchos otros). En este sentido, entender la dinámica del criptomercado de drogas ilegales también da luces sobre el funcionamiento de otros criptomercados ilegales y la manera como deben confrontarlos las autoridades de control.

\section{Conclusiones}

En este artículo se propuso un nuevo modelo analítico para entender las ventas de drogas ilegales en la web oscura en el contexto del criptomercado de drogas ilegales. El modelo se basa en el modelo de competencia monopolística con externalidades en red expuesto por Belleflamme y Peitz (2010, cap. 20), quienes a su vez se basan en el modelo canónico de Dixit y Stiglitz (1977).

El modelo muestra que las políticas de interdicción en esta clase de mercados tienden a ser contrarrestadas por la entrada de nuevos vendedores, y la consecuente apertura de nuevas páginas web de ventas de drogas en la red oscura. En concreto, se mostró que con una elasticidad-precio de demanda constante para cada una de las páginas web, un incremento en la probabilidad de interdicción conduce a alzas en los precios de equilibrio (de corto y largo plazo) de las variedades de drogas-páginas web, pero tiene un efecto neto nulo sobre el nivel de ventas de equilibrio.

Adicionalmente, debido a la presencia de efectos de externalidad en red en el consumo de drogas online, el endurecimiento de las políticas de interdicción no solo lleva a un in- 
cremento de la competencia que induce a la apertura de nuevas páginas web, sino que produce un efecto de feedback que lleva a que un mayor número efectivo de consumidores decida adquirir los dispositivos para acceder a las plataformas de drogas en la web oscura, y de esa forma comprar drogas ilegales. Este efecto de retroacción positiva implica que este criptomercado presente resiliencia frente a las políticas de control y devela la adaptabilidad estratégica de los traficantes bajo esta nueva modalidad de ventas ilegales.

En este escenario, las autoridades de control enfrentan nuevos retos frente al control y persecución de las drogas ilegales. Sin duda, estas deben adquirir nuevas y cada vez más sofisticadas herramientas tecnológicas para hacer rastreo y detección de operaciones ilegales en la web oscura, así como desarrollar cada vez más destrezas para entender la dinámica del criptomercado y la versatilidad de los traficantes en este contexto. El modelo analítico propuesto implica que las autoridades deben detectar y cerrar páginas web encriptadas de forma eficiente, pero al mismo tiempo desarrollar los mecanismos informáticos que impidan a los vendedores migrar en poco tiempo a otras páginas web. Esto sugiere identificar rápidamente las posibles rutas de migración a otras páginas web así como desarrollar algoritmos de bloqueo de estas en un tiempo mínimo.

Lo anterior implica que los gobiernos deben hacer mayores niveles de inversión en investigación y desarrollo para consolidar las labores de inteligencia necesarias para contrarrestar estas nuevas formas criminales en ascenso. De hecho, el avance tecnológico de punta de los cibercriminales implica que los gobiernos deberían mantener niveles de inversión constantes o crecientes en inteligencia policiaca especializada en esta clase de actividades. En este escenario, cobran cada vez más importancia las labores de inteligencia y contra-inteligencia, y se vuelven más relevantes que el uso de la fuerza tradicional para contener la violencia y mantener el orden, tal como se hace en los mercados de drogas tradicionales. De ahí la importancia de sostener incentivos monetarios suficientes para el desarrollo de investigación criminalística de punta así como de personal altamente calificado.

Estas implicaciones para la política criminal son mucho más relevantes en la coyuntura actual de pandemia provocada por la Covid-19, la cual ha incrementado significativamente todas las actividades en Internet, incluyendo las operaciones y transacciones ilegales en los criptomercados ${ }^{10}$.

De hecho, la pandemia ha llevado a un proceso de reingeniería de diversas actividades criminales, las cuales han tendido a tecnificarse mucho más y a orientarse en mayor medida hacia la comisión de fraudes electrónicos, la realización de transacciones en la web oscura, y la implementación de formas más sofisticadas de lavado de activos y estafas a través de esquemas piramidales tipo Ponzi. La política criminal no debe quedarse atrás: debe también desarrollar nuevos procesos de inteligencia de punta ligados al manejo del big data, la analítica de datos online, y al rastreo de actividades sospechosas en la red profunda y en la red oscura, guiados por la criminología de frontera, la ingeniería de sistemas, el machine learning y, por supuesto, los nuevos desarrollos en la economía del crimen.

10 Durante las semanas posteriores al inicio de la pandemia -luego del 21 de marzo de 2020-, el porcentaje de transacciones exitosas o con problemas en la red oscura con respecto al total declinó considerablemente pasando de porcentajes entre el 60 y el $100 \%$ a un porcentaje del $21 \%$, como consecuencia del choque causado por la cuarentena decretada en la mayor parte de los países en que se concentran las transacciones del criptomercado (Bergeron et al., 2020). No obstante, a lo largo del mismo año hay evidencia de que el volumen de comercio transado en diversos criptomercados ascendió progresivamente. Para finales de mayo ya había un ligero incremento de la actividad del criptomercado en la Dark Web de la mano de una baja en los precios de las ventas al por mayor (CIDE, 2020; EMCCDA, 2020). 
Sociedad y Economía N 44 (Sep - dic 2021) / e-ISSN: 2389-9050 / e10610962

Competencia monopolística y externalidades en el criptomercado de drogas

\section{Referencias}

Ballester, C., Calvó-Armengol, A. y Zenou, Y. (2006). Who's who in networks. Wanted: The key player. Econometrica, 74(5), 1403-1417. https://doi.org/10.1111/j.1468-0262.2006.00709.x

Ballester, C., Zenou, Y. y Calvó-Armengol, A. (2010). Delinquent networks. Journal of the European Economic Association, 8(1), 34-61. https://doi.org/10.1111/j.1542-4774.2010.tb00494.x

Belleflamme, P. y Peitz, M. (2010). Industrial organization: markets and strategies. Cambridge University Press.

Bergeron, A., Décary-Hétu, D. y Giommoni, L. (2020). Preliminary findings of the impact of COVID-19 on drugs crypto markets. International Journal of Drug Policy, 83, 102870. https://doi.org/10.1016/j. drugpo.2020.102870

CIDE. (2020). El nuevo mercado de las drogas en tiempos del COVID-19: ¿Qué cambios se pueden esperar? https://www.cide.edu/coronavirus/2020/05/19/el-nuevo-mercado-de-las-drogas-en-tiempos-del-covid19-que-cambios-se-pueden-esperar/

Décary-Hétu, D., Paquet-Clouston, M. y Aldridge, J. (2016). Going international? Risk taking by cryptomarket drug vendors. International Journal of Drug Policy, 35, 69-76. https://doi.org/10.1016/j. drugpo.2016.06.003

De-León, I. y Salcedo, E. (2014). El crimen como oficio. Ensayos sobre economía del crimen en Colombia. Universidad Externado de Colombia.

Dixit, A. K. y Stiglitz, J. E. (1977). Monopolistic competition and optimum product diversity. The American economic review, 67(3), 297-308. https://www.jstor.org/stable/1831401

Dolliver, D. S. (2015). Evaluating drug trafficking on the Tor Network: Silk Road 2, the sequel. International Journal of Drug Policy, 26(11), 1113-1123. https://doi.org/10.1016/j.drugpo.2015.01.008

Duncan, G. (2014). Más que plata o plomo. El poder político del narcotráfico en Colombia y México. Penguin Random House Grupo Editorial.

El Tiempo. (2018). Cómo operaba la red que vendía drogas en colegios por WhatsApp. https://www.eltiempo. com/justicia/delitos/como-operaba-la-banda-que-vendia-drogas-en-colegios-por-whatsapp-263774

EMCCDA -European Monitoring Centre for Drugs and Drug Addiction-. (2020). Special Report. COVID-19 AND DRUGS I Drug supply via darknet markets. https://www.emcdda.europa.eu/system/files/ publications/13042/EMCDDA-report_COVID19-darknet-final.pdf

García-Sigman, L. I. (2017). Narcotráfico en la Darkweb: los criptomercados. URVIO Revista Latinoamericana de Estudios de Seguridad, (21), 191-206. http://dx.doi.org/10.17141/urvio.21.2017.2824

Garoupa, N. (1997). The theory of optimal law enforcement. Journal of economic surveys, 11(3), 267-295. https://doi.org/10.1111/1467-6419.00034

Hardy, R. A. y Norgaard, J. R. (2015). Reputation in the Internet black market: an empirical and theoretical analysis of the Deep Web. Journal of Institutional Economics, 12(3), 515-539. https://doi.org/10.1017/ S1744137415000454

Heber, A. (2009). The Networks of Drug Offenders. Trends in Organized Crime, 12, 1-20. https://doi. org/10.1007/s12117-008-9055-8

Janetos, N. y Tilly, J. (2017). Reputation Dynamics in a Market for Illicit Drugs. Cornell University. https:// arxiv.org/pdf/1 703.01937.pdf

Kruithof, K., Aldrige, J., Décary-Hétu, D., Sim, M., Dujso, E. y Hooners, S. (2016). Internet facilitated drug trade. An analysis of the size, scope and the role of the Netherlands. RAND. 
Sociedad y Economía N 44 (Sep - dic 2021) / e-ISSN: 2389-9050 / e10610962

Leonardo Raffo, Diego Gómez-Calderón y Carolina Ramírez-Andrade

Malm, A. y Bichler, G. (2011). Networks of Collaborating Criminals: Assessing the Structural Vulnerability of Drug Markets. Journal of research in crime and Delinquency, 48(2), 271-297. https://doi. org/10.1177/0022427810391535

Martin, J. (2014). Drugs on the dark net: How cryptomarkets are transforming the global trade in illicit drugs. Springer Verlag.

Polinsky, A. M. y Shavell, S. (2000). The economic theory of public enforcement of law. Journal of economic literature, 38(1), 45-76. https://doi.org/10.1257/jel.38.1.45

Raffo, L. (2016). Notas de clase curso Microeconomía Il, Maestría en Economía Aplicada (documento inédito). Universidad del Valle.

Raffo, L. y Gómez, D. (2017). Redes criminales y corrupción en la era del microtráfico y el narcomenudeo. Revista de Economía Institucional, 19(37), 227-261. https://doi.org/10.18601/01245996.v19n37.12

Raffo, L. y Segura, J. L. (2015). Las redes del narcotráfico y sus interacciones: un modelo teórico. Revista de Economía Institucional, 17(32), 183-212. https://doi.org/10.18601/01245996.v17n32.06

Raffo-López, L. y Segura, J. L. (2018). La ineficacia de las políticas de represión a la oferta de drogas: una explicación alternativa. Ensayos de economía, 28(52), 165-201. https://doi.org/10.15446/ede. v28n52.71862

The Economist. (2016). Shedding light on the dark web. http://www.economist.com/news/ international/21702176-drug-trade-moving-street-online-cryptomarkets-forced-compete

Tirole, J. y Rochet, J. C. (2003). Platform competition in two-sided markets. Journal of the european economic association, 1(4), 990-1029. https://doi.org/10.1162/154247603322493212 